\title{
The Formation of Elements
}

\author{
Zhe Yin \\ Department of Mathematics, Yanbian University, China
}

\begin{abstract}
In this paper, we proposed the smallest particles of the composition of the earth, the role of hydrogen atoms, according to the gravitational wave theorem, the variable field theorem and mathematical modeling analysis. The roles and relations of protons and neutrons are elucidated. Any material is a combination of hydrogen atoms, and finally three kinds of material basis which form the earth were prompted. New principle of inter atomic periodic law was proposed.
\end{abstract}

Keywords: element; substance; gravitational wave;

\section{Introduction}

The universe is an energetic field filled with energy, and the cosmic galaxy is the geometric equilibrium of the energy set $[1,2,3]$. Each planet is a dynamic motion body of proton group, which tends to spherical through the rotation and revolution [3]. The planet which has the largest energy transfer the energy through the gravitational wave[10] in energy field, also the variable force field. So that the density of the planets which have the small energy gradually increased. And a substance which has a small molecular weight gradually becomes a substance having a large molecular weight, and so on.

We proposed in 2010 that substances change to the direction of increase of atomic weight and molecular weight [1]. And we proposed that spiral trajectory of gravitational waves [4] and the variable force field is the condition of energy conversion in February 2016[5,6]. We also proposed that the motion trajectory of the electrons, proposed the law of motion that, spiral trajectory gravitational wave produces entanglement in the process of convergence and divergence, increases the energy, in the trajectory of Mobius', and spiral gravitational wave convergences inwards in March 2016 [5,6,10]. Inward convergence is equivalent that the centripetal force is greater than buoyancy and other external field forces, outward divergence equivalent buoyancy and other external field force is greater than the centripetal force.

\section{Theoretical Preparation And Definition Preparation[11]}

\subsection{Generation and propagation trajectory theorem of gravitational waves}

Theorem 1: (Gravitational waves existence theory) [5,7,8,9,10,11]: There are A, B two points. A is the wave source of gravitational field, and B is a point in the gravitational field. The existence of energy rotational motion (including proton, neutron, atomic nucleus or planet) at $\mathrm{A}$ is the necessary and sufficient condition of the existence of gravitational waves at $\mathrm{B}$. Direction is the bidirection of the path tangent at point $\mathrm{B}$, and the limit of the convergence direction is A point.

Prove: Let say the distance of A and B is r, if A, B are stationary points, it only exists the physical factor of "distance $r$ ", do not form elements of waves. Only the rotational movement can generate speed and energy. That is the speed of B point $V b$ and the angular velocity of A point $d \theta$ have a functional relationship.

$$
\begin{aligned}
& V b=f(d \theta, r),{ }_{\text {when time }} \Delta t \rightarrow 0, \text { angular velocity } \Delta \theta \rightarrow 0, \text { and } \\
& V b=\frac{d r}{d t}=\lim _{\Delta \theta \rightarrow 0} \frac{[f(\theta+\Delta \theta), r]-[f(\theta), r]}{\Delta \theta},
\end{aligned}
$$

We have a conclusion that $\mathrm{A}$ is the wave source of gravitation, and point $\mathrm{B}$ exists the gravitational wave emitted from wave source A.

2.2 Theorem 2(Gravitational wave stability theory) [5,7,8,9,10,11]: A is a gravitational wave source, B is a point in gravitational field of A. The necessary and sufficient conditions of stable gravitational wave at B point is:

$$
\left.\frac{d r}{d \theta}=b \quad \text {, ( } \mathrm{b} \text { is a constant }\right) .
$$


Prove: According to Theory 1 ,

$V b=\frac{d r}{d t}=\lim _{\Delta \theta \rightarrow 0} \frac{[f(\theta+\Delta \theta), r]-[f(\theta), r]}{\Delta \theta}$,

The necessary and sufficient conditions of stable gravitational wave is that the speed of B point is stable;

The necessary and sufficient conditions of stable speed at B point are that the angular velocity of A point is stable;

The necessary and sufficient conditions of stable angular velocity of A point is that the angular velocity of A point and speed of B point is proportional.

That is,

$\frac{V b}{d \theta}=\frac{d r}{d \theta}=b \quad$, (b is a constant)

and,

$r=a+b \theta$,

formula (1) is the equations of gravitational waves, that is the track of gravitational waves.

Inference of theorem 2: If a gravitational wave track meet Archimedean spiral, this must be stable gravitational waves.

There is, $r=a+b \theta$.

$r$ is the distance of $A$ to $B$; $a$ is the spiral length of $A$ to $B$; $b$ is the distance between the spirals.

\subsection{Potential difference existence theorem}

In the relatively stable gravitational wave field, if there is a rotation of the line, then there exists the following theorem.

Theorem 3: The potentials at each point on an independent rotation line are equal and homomorphic.

In the relatively stable gravitational wave field, there are two different rays emanating from the same point A, each having two points $B$ and $C$, respectively.

Theorem 4: The two rays $\mathrm{AB}$ and $\mathrm{AC}$ at point $\mathrm{A}$ rotate at the same time in the clockwise (or anticlockwise) direction, and the potential difference between $\mathrm{A}$ and $\mathrm{B}$ is generated according to the entanglement of the helix trajectory and the electron. Similarly, a potential difference between A and C is also generated. And the entanglement force at point $\mathrm{A}$ and $\mathrm{B}$ is small.

Inference of theory 4: there is a plane gravitational wave field, then the maximum potential difference is the gradient direction of the gravitational wave center of the normal direction.

Theorem 5: In a relatively stable gravitational wave field region, filled with the same potential difference of homomorphic particles; then the region meets the uniform distribution of particles, and each particle potential difference in the same direction.

\subsection{Definition preparation}

We set an atom $\mathrm{s}$ as the origin of coordinates and establish two rectangular coordinates. One is the z-axis parallel to the earth's rotation axis of the earth rectangular coordinate system $\mathrm{x}, \mathrm{y}, \mathrm{z}$; the other is the earth revolution sun revolution plane sun coordinate system $\mathrm{p}, \mathrm{q}, \mathrm{r}$. The force of the earth's gravitational wave field is expressed by vector $\mathrm{x}, \mathrm{y}$ and $\mathrm{z}$, and the force of the atom $\mathrm{s}$ under the sun's gravitational wave field is denoted by vector $\mathrm{p}, \mathrm{q}$ and $\mathrm{r}$.

The atom $\mathrm{s}$ is in a relatively stable state. A coordinate system is established such that vector $\mathrm{x}, \mathrm{y}, \mathrm{z}, \mathrm{p}, \mathrm{q}, \mathrm{r}$, the six force variables are represented on the same coordinate system.

Definition 1: At the point of atom s, we establish a coordinate space, 4-dimensional 120-degree angular coordinate system, the coordinate origin of which is $0, \mathrm{H} 1, \mathrm{H} 2, \mathrm{H} 3, \mathrm{H} 4$. We call it 4-dimensional coordinate system $\mathrm{H}$.

\section{Element Composition Theorem of Zhe Yin}

The earth's rotation and revolution movement, let the earth tends to become sphere [1,2]. Moon does not rotate during lunar rotation of the earth's process, so the forces on the earth's particles do not consider the impact of the moon's rotating gravitational wave. According to reference[3], The universe is the relative steady state of the energy set of revolution or revolution. The galaxy's stars are all filled with electrons inside and outside the set of energy. In each star, the minimum atom is determined through the external gravitational wave 
field and the role of gravity (field the equilibrium state from the entanglement and deposition of electrons). The atomic weights of the smallest atoms of each star are different. The smallest atom of the earth is a hydrogen atom.

Theorem1: All the substances on earth are made up of different combinations of hydrogen atoms.

The combination of hydrogen atoms satisfies certain law, we study the composition of basis of the base of the hydrogen atom in the following. That is, the mathematical description of the gravitational wave.

\subsection{Mathematical model of combined gravitational force field}

We set the rectangular coordinates $\mathrm{x}, \mathrm{y}, \mathrm{z}$ on the earth and the rectangular coordinates $\mathrm{p}, \mathrm{q}, \mathrm{r}$ on the Earth's orbital plane. The particle $\mathrm{s}$ is the coordinate origin. There are the following variable field mathematical models.

$$
\begin{aligned}
& \left\{\begin{array}{c}
x^{\prime}(t)=a_{11} x+a_{12} y+a_{13} z+w_{1}(t) \\
y^{\prime}(t)=a_{21} x+a_{22} y+a_{23} z+w_{2}(t) \\
z^{\prime}(t)=a_{31} x+a_{32} y+a_{33} z+w_{3}(t)
\end{array}\right. \\
& \left\{\begin{array}{c}
p^{\prime}(t)=b_{11} p+b_{12} q+b_{13} r+e_{1}(t) \\
q^{\prime}(t)=b_{21} p+b_{22} q+b_{23} r+e_{2}(t) \\
r^{\prime}(t)=b_{31} p+b_{32} q+b_{33} r+e_{3}(t)
\end{array}\right.
\end{aligned}
$$

Formula(2) is the variable force field model in the rectangular coordinates on earth (referred to as the earth rectangular coordinates ), formula(3) is the variable force field model in the rectangular coordinates on the earth revolution plane(referred to as the sun rectangular coordinates). The variable force function is derivable because it is helix. The Taylor expansion or Lagrangian expansion can be used to elicit the above two mathematical models.

$\mathrm{x}, \mathrm{y}, \mathrm{z}$ is the force function of the particle $\mathrm{s}$ in the earth variable force field, in the earth rectangular coordinates. p,q, $\mathrm{r}$ is the force function of the particle $\mathrm{s}$ in the sun variable force field, in the sun rectangular coordinates.

$\mathrm{w}_{1}(\mathrm{t})$ is the nonlinear factor of the earth rectangular coordinates in the $\mathrm{x}$ - direction.

$w_{2}(t)$ is the nonlinear factor of the earth rectangular coordinates in the $y$-direction.

$\mathrm{w}_{3}(\mathrm{t})$ is the nonlinear factor of the earth rectangular coordinates in the $\mathrm{z}$-direction.

$\mathrm{e}_{1}(\mathrm{t})$ is the nonlinear factor of the sun rectangular coordinates in the $\mathrm{p}$ - direction.

$\mathrm{e}_{2}(\mathrm{t})$ is the nonlinear factor of the sun rectangular coordinates in the $\mathrm{q}$-direction.

$\mathrm{e}_{3}(\mathrm{t})$ is the nonlinear factor of the sun rectangular coordinates in the $\mathrm{r}-$ direction.

$\mathbf{t}$ is the time variable.

There is

$$
\begin{aligned}
& \frac{d}{d t}\left(\begin{array}{l}
x \\
y \\
z
\end{array}\right)=\left(\begin{array}{lll}
a_{11} & a_{12} & a_{13} \\
a_{21} & a_{22} & a_{23} \\
a_{31} & a_{32} & a_{33}
\end{array}\right)\left(\begin{array}{l}
x \\
y \\
z
\end{array}\right)+\left(\begin{array}{l}
w_{1} \\
w_{2} \\
w_{3}
\end{array}\right) \\
& \frac{d}{d t}\left(\begin{array}{l}
p_{q} \\
r
\end{array}\right)=\left(\begin{array}{lll}
b_{11} & b_{12} & b_{13} \\
b_{21} & b_{22} & b_{23} \\
b_{31} & b_{32} & b_{33}
\end{array}\right)\left(\begin{array}{l}
p_{1} \\
q \\
r
\end{array}\right)+\left(\begin{array}{l}
e_{1} \\
e_{2} \\
e_{3}
\end{array}\right)
\end{aligned}
$$

Among them, $A_{i j}=\left(\begin{array}{lll}a_{11} & a_{12} & a_{13} \\ a_{21} & a_{22} & a_{23} \\ a_{31} & a_{32} & a_{33}\end{array}\right), B_{i j}=\left(\begin{array}{lll}b_{11} & b_{12} & b_{13} \\ b_{21} & b_{22} & b_{23} \\ b_{31} & b_{32} & b_{33}\end{array}\right)$ is coefficient matrix.

There is function $\mathrm{G}$, which makes

$$
\left(\begin{array}{l}
x \\
y \\
z
\end{array}\right)=G\left(\begin{array}{l}
p \\
q \\
r
\end{array}\right)
$$

The variable force field is the premise of energy conversion and energy generation. Earth movement tends to sphere, so the limits of change of the coefficient matrix $A_{i j}$ and $B_{i j}$ is unit matrix. $(i=1,2,3 ; j=1,2,3)$

\subsection{4 - dimensional 120 - degree angular coordinate system $H$}

Theorem2: Any four tetrahedra of the four vertices must fall in the 4-dimensional 120-degree angular coordinate system $\mathrm{H}$ 's coordinate axis, through the rotation transformation of coordinate axis. 
Because the tetrahedron is bounded and the sum of the inner product of the six edge vectors is equal to zero, just as the three vertices of a triangle can fall on the coordinate axes of the three-dimensional rectangular coordinates through the rotation transformation of the coordinate axes.

The angle between earth's rotation axis and the ecliptic plane is 23 degrees 26 points, the earth's rotation coordinate system with the earth's revolution on the plane of the sun rectangular coordinate system between six axes, is always linearly independent. The six sub-vectors of the relative stable particle on the earth are bounded, and the sum of the sub-vectors is equal to zero.

Theorem 3: Earth has a relatively stable particle s, the force component of gravitational wave force of s particle on the earth rectangular coordinates xyz is the vector ox, vector oy, and vector oz; the force component of gravitational wave force of $\mathrm{s}$ particle on the sun rectangular coordinates pqr is the vector op, vector oq, and vector or. There are $\mathrm{A}, \mathrm{B}, \mathrm{C}$ and $\mathrm{D}$ points on each coordinate axis of the coordinate system of the four-dimensional 120-degree angle coordinate system $\mathrm{H}(\mathrm{H} 1, \mathrm{H} 2, \mathrm{H} 3, \mathrm{H} 4)$ as following:

vector $\mathrm{ox}=$ vector $\mathrm{OA}-$ vector $\mathrm{OB}$;

vector $\mathrm{oy}=$ vector $\mathrm{OA}-$ vector $\mathrm{OC}$;

vector $\mathrm{oz}=$ vector $\mathrm{OA}-$ vector $\mathrm{OD}$;

vector $\mathrm{op}=$ vector $\mathrm{OB}-$ vector $\mathrm{OC}$;

vector oq= vector $\mathrm{OB}-$ vector $\mathrm{OD}$;

vector or= vector $\mathrm{OC}-$ vector $\mathrm{OD}$;

And, because

vector $\mathrm{ox}+$ vector $\mathrm{oy}+$ vector $\mathrm{oz}+$ vector $\mathrm{op}+$ vector $\mathrm{oq}+$ vector or $=$ vector 0 ,

so there exists

$($ vector $\mathrm{OA}$ - vector $\mathrm{OB})+($ vector $\mathrm{OA}-$ vector $\mathrm{OC})+($ vector $\mathrm{OA}-$ vector $\mathrm{OD})$

$+($ vector OB- vector OC $)+($ vector OB- vector OD $)+($ vector OC- vector OD $)=$ vector 0 .

Definition 2: The four unit vectors $\mathrm{h} 1, \mathrm{~h} 2, \mathrm{~h} 3, \mathrm{~h} 4$ on the $\mathrm{H}$ coordinate system are the basic basis of the description of the force vector representation of the steady state of the earth.

Theorem 4: The basic structure of atoms on the earth is based on the basic basis of the coefficient of composition times, tetrahedral structure of the combination. Four tetrahedrons correspond to neutrons and six boundaries to protons.

The three particles (three hydrogen atoms) between the two vertices of the tetrahedron are linearly arranged. In the combined gravitational wave field of the earth and the sun, electrons rotate linearly in a spiral motion. The particle in the middle must be charged, that is protons (there is potential difference). According to the existence theorem of potential difference 5, in a region of homomorphic gravitational wave field filled with homomorphic particles with the same potential difference, the particles in the region are uniformly distributed and the direction of potential difference of each particle is the same. Therefore, there is only one state (the same potential) between the four vertices of the tetrahedron and the adjacent vertices of other atoms. This node has no potential difference, that is, neutrons. Neutron equivalent to the reservoir, the proton is equivalent to the river. Neutrons can overlap, with potential capacity, play the role of storage power. Proton has a potential difference, play a role in the production of electricity.

Theorem 5 (Theorem of neutron): Neutrons have the role of electronic energy storage, and overflow when they are fully charged.

\subsection{The composition of substance}

Definition 3: The basic basis block is defined as a linear combination of six edge vectors of one tetrahedron, and six protons correspondence;

The second basic base block is defined as a linear combination of nine edge vectors of two tetrahedrons, and nine protons correspondence

The third basic base block is defined as a linear combination of 10 edge vectors of two tetrahedrons, and 10 protons correspondence(Four corners, plus two diagonal).

Theorem 6: All the atomic structures of the earth's matter have the following general expression:

The number of protons (the number of nuclear charges) of an atom consisting of the basic base block: $\mathbf{K 1}=\mathbf{6 m}$ $+\mathbf{n}$. Here $\mathrm{n}$ is a natural number from 0 to 5 and represents the number of residual charges other than the basic base block, and $\mathrm{m}$ is a natural number representing the number of the base block tetrahedra.

The number of protons (the number of nuclear charges) of an atom consisting of the second basic base block: $\mathbf{K} \mathbf{2}=\mathbf{9 m}+\mathbf{n}$. Here $\mathrm{n}$ is a natural number from 0 to 8 and represents the number of residual charges other than the second basic base block, and $\mathrm{m}$ is a natural number representing the number of the second basic base block 
The number of protons (the number of nuclear charges) of an atom consisting of the third basic base block: K3 $=\mathbf{1 0} \mathbf{m}+\mathbf{n}$. Here $\mathrm{n}$ is a natural number from 0 to 9 and represents the number of residual charges other than the third basic base block, and $\mathrm{m}$ is a natural number representing the number of the third basic base block

Inference of theorem 6: According to the differences of symmetry and stability of the atomic structure, the isotopes of the atoms composed of the second and third base blocks are more than the isotopes of the atoms composed of the basic base block isotopes.

Theorem 7(The theorem of the electric field around atom): The residual charge of the steady state atom is in the space around the atom, forming the following 6 kinds of state of power intensity ratio (basic basic block as an example): $0,+1-5,+2-4,+3-3,+4-2,+5-1$. Divide the space around the atom according to the positive and negative power intensity ratio. The sum of the positive charges is equal to the sum of the negative charges. The smaller the absolute value of power intensity ratio is, the stronger the electric power is, and the smaller the surrounding space occupies. The larger the absolute value of power intensity ratio is, the weaker the electric power is and the bigger the surrounding space occupies. The entanglement strength is inversely proportional to the occupied space.

Description: "0" is equal to not charged, "+1 -5 "is equal with has 1 strong electricity and 5 weak -1 electricity; “+2 -4”'is equal with has 2 strong electricity and 4 weak -1 electricity; “+3 -3"is equal with has 3 strong electricity and 3 weak -1 electricity; “+4 -2"is equal with has 4 strong electricity and 2 weak -1 electricity; " +5 -1 '” equal with has 5 strong electricity and 1 weak -1 electricity.

Theorem 8 (law of the periodic nature of the atomic properties): the number of charges other than basis base blocks of atom determines the periodic nature of the atom. If the residual charge is same, then they are the same family or have similar nature.

Theorem 9: The atoms of element numbers 5 to 12 in the periodic table are composed of the third basic base block $(\mathbf{K 3}=\mathbf{1 0 m}+\mathbf{n})$. In addition, the atoms of element numbers 1 to 56 are composed of basic base blocks $(\mathbf{K} \mathbf{1}=\mathbf{6 m + n})$. The lanthanide atoms of elements numbers 57 to 71 consist of $\mathrm{k} 1$ and $\mathrm{k} 2$ product union bases $(\mathbf{K} 4$ $=\mathbf{k} 1 \times \mathbf{k} 2+\mathbf{3}+\mathbf{n}, \mathbf{n}=\mathbf{0 - 1 4})$, and the number of lanthanide atoms is equal to 15 , because $(\mathrm{k} 1+\mathrm{k} 2=6+9=15)$ is also very natural, lanthanide first atom residual charge is equal to 3 is also normal. element number 72 to 88 atoms are the sum of the product of $\mathrm{k} 1, \mathrm{k} 2$ and $\mathrm{k} 3,(\mathbf{K 5}=\mathbf{5 k} \mathbf{1}+\mathbf{2} \mathbf{k} \mathbf{2}+\mathbf{2} \mathbf{k} \mathbf{3}+\mathbf{4}+\mathbf{n}, \mathbf{n}=\mathbf{0 - 1 6})$. And the number of remaining charges of the first atom equal to 4 is also normal. the actinide atom of the elements number 89 to 103 is the sum of the product of $\mathrm{k} 1, \mathrm{k} 2$ and $\mathrm{k} 3,(\mathbf{K} \mathbf{6}=\mathbf{k} \mathbf{1} \times \mathbf{k} \mathbf{2}+\mathbf{2} \mathbf{k} \mathbf{3}+\mathbf{2} \mathbf{k} \mathbf{1 + 3 + n}, \mathbf{n}=\mathbf{0}-\mathbf{1 4})$ the actinides are equal to 15 , because $(\mathrm{k} 1+\mathrm{k} 2=6+9=15)$ is also very natural. The atomic number of the element number 104 to 118 is

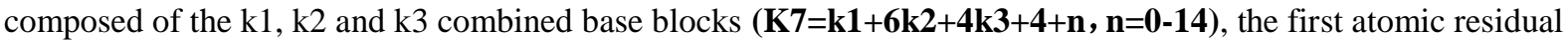
charge number is equal to 4 is also normal.

Relative to metal atoms, nonmetallic atoms have the relatively small number of charges in the basis of the basic block within, resulting in the formation of negative charge around the field strength. But the cohesion between the basic blocks between the atoms is not strong, highlighting the gravitational wave field and the sun's gravitational wave field strength of the gap. That leads to the degradation of the 4-dimensional 120-degree angular coordinate system. The third base block structure tends to be planar structure.

Theorem 10 (non-metal theorem): the non-metallic atoms of the third basic block of internal particles, the asymmetric unstable force conditions, and gradually meet the four-dimensional 120-degree angular coordinate system degenerate into three-dimensional 120-degree angular coordinates, or a planar six-dimensional 60 -degree angular coordinate system. And the sum of the inner products of the six force components is equal to zero. Space tetrahedron basic base block structure gradually tends to be planar hexagonal structure.

For example, graphite is a non-metallic carbon structure for the layered hexagonal and diamond is a metal hexahedron spatial structure of carbon. Non-metallic liquid closed curing (to reduce the role of the earth's gravitational waves), could acquire metal state crystals.

\section{Conclusion}

In this paper, the composition of the earth's matter is solved and the relationship between protons and neutrons is clarified. The substance is a combination of hydrogen atoms, and three basic blocks of earth material are proposed. A new inter atomic periodic law is proposed. And the theoretical basis for the future discovery and manufacture of new elements and different forms were proposed. In this paper, the carbonation of water, the research of the real role of $(\mathrm{OH})$ will play a role. The structural stratification and multi-neutralization is the best choice in the process of storage battery selection. 


\section{References}

[1]. Zhe Yin,(2010).The Co-Planarity and Symmetry Principle of Earthquake occurrence, International Journal of Geosciences,pp:38-43.

[2]. Zhe Yin, Yunfei Guo, Maosheng Lai,(2011),Analysis of Climatic Change from Energy Perspective, Procedia Environmental Science,Vol.11,Part A,pp:245-249.

[3]. Zhe Yin,dongxu liu.(2016). Analysis of the Relation between Sun Motion Law and the Change of Earth's Environment. International Journal of Geosciences, 7,249-256.

[4]. j. Weber, $(1959,2016)$, Gravitational Wave, Laser interferometer gravitational wave observatory, 2016.2.11.23:30.

[5]. Zhe Yin,dongxu liu.(2016). Electron trajectory, International Journal of Applied Science and Mathematics,3( 2),56-57.

[6]. Zhe Yin, Dongxu L (2016). Causes of sound wave on the moon. Int. J. Eng. Appl. Sci. 3(2):56-58.

[7]. Zhe Yin (Yin Z), Zhang W, Bie H (2016). Zhe yin's Theorem of Molecular Biology. Acad. J. Sci. Res. 4(4): 099-102.

[8]. Zhe Yin,(2016). Ecological Research, International Journal of New Technology and research (IJNTR)ISSN:2454-4116, Volume-2, Issue-3, March 2016 Pages 64-66.

[9]. Zhe Yin,(2016). All chemical reactions is change of electronic entanglement in the way, International Journal of Engineering and Applied Sciences (IJEAS)ISSN: 2394-3661,3(3):20-21.

[10]. Zhe Yin,(2016). Zhe yin's energy and Wave theorem, International Journal of Engineering and Advanced Research Technology (IJEART)ISSN: 2454-9290, 2(3):44-45.

[11]. Zhe Yin.2017, The Function And Relation of Proton And Neutron. Int J Recent Sci Res. 8(1), pp. 15321-15324 